\title{
History of brain tumor surgery
}

\author{
Mark C. Preul, M.D. \\ Division of Neurological Surgery,Barrow Neurological Institute, St. Joseph's Hospital and Medical \\ Center, Phoenix, Arizona
}

The history of brain tumor surgery is central to the development of neurosurgery as a specialty. William Macewen is considered to have performed the first successful brain tumor removal in 1879 in a young woman. In his article he stated that he removed "the usual fungous tumor of the dura mater," likely a meningioma, after localizing the tumor to the region of the left central area. His comments imply that he had some experience with such tumors, although he does not mention prior successful surgical removals.

In the late 19th century accounts began to be published on attempts to remove brain tumors (meningiomas). Macewen, Victor Horsley, and William W. Keen began to perform aggressive maneuvers in attempting brain tumor removal. Nevertheless, they describe limited systematic diagnostic processes beyond localization by clinical examination.

Harvey Cushing is usually credited with making the greatest early strides toward brain tumor removal. Certainly, he did advance operative techniques with his adoption of new anesthetic methods and his use of "motor driven suction" and "electrosurgical devices." Cushing did not, however, take advantage of new technology for diagnosis in some critical instances. For example, he was slow to adopt Walter Dandy's ventriculography procedure. Although Dandy claimed that at least one third of brain masses could be readily localized using his air injection method, still in 1927 Cushing only advocated the method in "obscure cases."

Other practitioners were rapidly adopting new technology for use in neurosurgery. At least by the first decade of the 20th century, only a few years after the introduction to the world of x-rays in late 1895, Fedor Krause was using Xrays routinely for assistance in localizing intracranial tu- mors. In Cushing's 1913 chapter of W. W. Keen's textbook on surgery, the word " $\mathrm{x}$-rays" is used only once. In contrast, by 1911 Krause had written an entire chapter devoted to "Radiography," in which he promoted the benefits of $\mathrm{x}$ rays for diagnosis of masses and tumors that had changed the contours of the skull or that had left osseous deposits, and in cases in which the neurological examination was "mute."

The history of brain tumor surgery makes for fascinating reading. Advancements in technologies directly affected the ability of neurosurgeons to remove tumors and at the same time decreased operative risk. As well, the personalities of the first and second generations of neurosurgeons helped to forge through what were often discouraging surgical outcomes. At times their personalities mingled, providing for even more drama. Although we have made much progress in technological and diagnostic developments for neurosurgery, we still face the challenge of substantially increasing the survival rates for patients with many types of brain tumors.

I hope that the reader finds this issue of Neurosurgical Focus informative and entertaining. The goal was not to promote dry overviews of the subject, but to provide more in-depth analyses of various subtopics within the history and development of surgery for brain tumors. I am indebted to the authors, who took much time to research, write, and submit a group of high-quality articles. I am especially thankful for the efforts of my two coeditors, Drs. Samuel Greenblatt and Michael Schulder, whose backgrounds in the history of medicine and neurosurgical history made this issue possible. As always, Susan Lanterman and the staff of Neurosurgical Focus at the Journal of Neurosurgery have provided the epitome of publication support. 